\title{
Os fios de sutura cirúrgica e a enfermeira de centro cirúrgico: critérios de previsão e provisão segundo a natureza das instituições hospitalares*
}

\author{
SUTURES AND THE OPERATING ROOM NURSE: CRITERIA IN FORECASTING THE NEED FOR SUTURES \\ AND PROVISIONING ACCORDING TO NATURE OF THE HOSPITAL INSTITUTION
}

\author{
LOS HILOS DE SUTURA QUIRÚRGICAS Y LA ENFERMERA DE CENTRO QUIRÚRGICO: CRITERIOS DE \\ PREVISIÓN Y PROVISIÓN SEGÚN LANATURALEZA DE LAS INSTITUCIONES HOSPITALARIAS
}

\section{Anita Romano Ribeiro1, Kazuko Uchikawa Graziano²}

\author{
RESUMO \\ O estudo identificoue \\ descreveu os critérios \\ adotados pelas enfermeiras \\ na seleção dos fios de sutura \\ cirúrgica para a elaboração \\ da sua previsão e posterior \\ provisão das Unidades de \\ Centro Cirúrgico, segundo a \\ natureza pública ou privada \\ das instituições hospitalares. \\ Os dados foram coletados \\ por meio de entrevista \\ estruturada e participaram do \\ estudo 74 hospitais do \\ município de São Paulo. A \\ maioria dos hospitais \\ pertence à rede privada e de \\ porte médio, com Centro \\ Cirúrgico geral e até 500 \\ procedimentos/mês. A \\ natureza determina a \\ modalidade de aquisição, \\ tipos de procedimentos \\ cirúrgicos e a variedade de \\ fios adquiridos. As \\ enfermeiras participam dos \\ processos de aquisição, \\ distribuição e utilização de \\ fios de sutura.
}

\section{PALAVRAS-CHAVE}

Enfermagem de Centro Cirúrgico. Suturas. Armazenamento de materiais e provisões.

Centro Cirúrgico hospitalar.

\begin{abstract}
The study has identified and described criteria adopted by the nurses in selecting and calculating quantities of surgical sutures to supply the Surgical Center, according to the public or private nature of the surgical centers. The data were collected using structured questionnaires in 74 hospitals within the municipality of São Paulo. The majority of hospitals are medium in size and privately owned with a general Operation Room and up to 500 surgical procedures performed per month. The procedures, the type and the variety of surgical sutures are related to the nature of hospital management.

However, there are no criteria to select surgical sutures and their distribution in the operation rooms. The nurses participate on the procedures for purchasing, distribution and use of the surgical sutures.
\end{abstract}

\section{KEYWORDS}

Operating room nursing.

Sutures.

Materials and supplies stockpiling.

Surgery department hospital.

\section{RESUMEN}

El estudio identificó y describió los criterios adoptados por las enfermeras al seleccionar los hilos de sutura quirúrgica para hacer la planificacióny posterior adquisición de los mismos para cada unidad de Cirugía, con variables según el tipo de institución hospitalaria, ya sea pública o privada. Los datos fueron obtenidos por medio de entrevistas estructuradas realizadas en 74 hospitales del municipio del Estado de São Paulo, Brasil. La mayoría de los hospitales de la red privada se clasifican como de porte medio, con una unidad de Cirugía General y hasta 500 procedimientos por mes. La naturaleza de la Institución determina la modalidad de adquisición, tipos de procedimientos quirúrgicos y la variedad de hilos de sutura adquiridos. Las enfermeras participan de los procesos de adquisición, distribución y uso de los hilos de sutura.

\section{PALABRAS CLAVE}

Enfermería en Centro Quirúrgico. Suturas. Almacenamiento de materiales y suministros. Servicio de cirurgia en hospital

\footnotetext{
* Extraído da Dissertação "Os fios de sutura cirúrgica e a enfemeira de centro cirúrgico: critérios de previsão e provisão segundo a natureza pública ou privada das instituições hospitalares." apresentada ao Programa de PósGraduação da Escola de Enfermagem da USP (EEUSP), 2001. Área de concentração Fundamentos de Enfermagem.

1 Enfermeira. Mestre em Enfermagem pela EEUSP.

aribeiro@medbr.jnj.com

2 Enfermeira. Professora Livre-Docente do Departamento de Enfermagem MédicoCirúrgica da EEUSP. rwgraziano@uol.com.br
} 


\section{INTRODUÇÃO}

Os fios de sutura cirúrgica foram conceituados por Goffi; Tolosa ${ }^{(1)}$ como materiais utilizados para selar vasos sangüíneos e aproximar tecidos, em ações de ligar e suturar. Surgiram e foram desenvolvidos ao longo dos séculos em função da necessidade de controlar hemorragias e também de favorecer a cicatrização de ferimentos ou incisões por primeira intenção.

Desde a antiguidade, um grande número de materiais de sutura foi testado e utilizado, tais como fibras vegetais, tendões, intestinos de vários animais, crina de cavalo, filamentos de ouro, dentre outros. Uma das menções mais antigas ao ato de suturar está registrada no papiro egípcio de Edwin Smith, que data de 3.500 a.C. O conceito de ligadura e sutura está também registrado nos escritos de Hipócrates e Galeno. Atribui-se ao médico árabe Rhazes a introdução da palavra kitgut, em 900 d.C., para designar fios confeccionados com tiras do intestino de animais herbívoros, utilizados como cordas de instrumentos musicais (kit) e largamente aplicados como sutura. Acredita-se que essa seja a origem da palavra Catgut, que denomina o fio de sutura cirúrgica mais conhecido em todos os tempos ${ }^{(2)}$.

Muitos séculos se passaram e persistiu a idéia de que toda ferida deveria ser estimulada a formar pus, para que ocorresse a cicatrização. Ambroise Paré, no século XVI, foi um dos primeiros cirurgiões a acreditar na capacidade de regeneração dos tecidos vivos. Introduziu fitas adesivas para coaptar bordas, além de difundir a ligadura, em substituição à cauterização com azeite fervente. No século XIX, o médico americano Philipe S. Physick, através de seus experimentos com suturas, admitiu a possibilidade de um fio que cumprisse sua função e depois desaparecesse, sendo absorvido pelos tecidos circundantes. Ao final do mesmo século, Joseph J. Lister introduziu métodos para redução da infecção cirúrgica, inclusive a desinfecção dos fios em solução de ácido carbólico ou fenol. Ele foi também pioneiro na utilização de Ácido Crômico para aumentar a resistência do Catgut à absorção. Com o advento da industrialização, materiais como a Seda e o Algodão tiveram seu uso difundido e passaram a ser anexados em agulha. Em 1900, já se dispunha de Catgut em tubo de vidro mergulhado em solução esterilizante. A partir da Primeira Guerra Mundial foram sendo desenvolvidos métodos de esterilização mais seguros, como o Cobalto 60, além de materiais sintéticos para a confecção dos fios de sutura cirúrgica. Por volta de 1940 começou a utilização da Poliamida e Poliéster, em 1962 do Polipropileno e a partir de 1970 os primeiros fios absorvíveis de origem sintética começaram a ser comercializados. Assim começou a tendência de se utilizar uma variedade de fios para sutura cirúrgica esterilizados, com agulhas pré-instaladas e fornecidos para pronto uso ${ }^{(2)}$.

Os fios utilizados para sutura e ligadura cirúrgica estão divididos em 2 grandes grupos: absorvíveis e inabsorvíveis. Os fios absorvíveis perdem gradualmente sua resistência à tração até serem fagocitados ou hidrolisados. Eles podem ser de origem animal (Catgut Simples e Cromado) ou sintéticos multi ou monofilamentares (Poliglactina, Poliglecaprone e Polidioxanona). Os fios inabsorvíveis se mantém no tecido onde foram implantados e podem ser de origem animal (Seda), mineral (Aço), vegetal (Algodão ou Linho) ou sintéticos (Poliamida, Poliéster, Polipropileno). Suas características e propriedades são definidas por órgãos oficiais e associações normatizadoras. No Brasil temos a Farmacopéia Brasileira ${ }^{(3)}$ e a norma brasileira NBR13904 da Associação Brasileira de Normas Técnicas ${ }^{(4)}$, dentre outras que se referem aos processos industriais.

Em cada sutura realizada, de acordo com o tipo de tecido ou a estrutura onde o material está sendo implantado e as particularidades do paciente, o cirurgião busca encontrar o Fio Ideal. Hering ${ }^{(5)}$ aponta características, tais como:

- alta resistência à ruptura permitindo o uso de diâmetros menores;

- boa segurança do nó;

- baixa reação tecidual;

- não favorecimento da instalação ou continuidade de um processo infeccioso;

- manutenção das bordas da incisão aproximadas até a fase proliferativa da cicatrização;

- boa visualização no campo operatório;

- desaparecer do tecido onde foi implantado, quando não for mais necessário. 
Dentro da variedade de fios cirúrgicos para ligadura e para sutura, não há como determinar um único fio, como fio ideal, uma vez que os tecidos a serem suturados possuem características morfológicas e funcionais distintas. Trabalhos como o de Benicewicz, Hopper ${ }^{(6)}$ consideram que o cirurgião escolhe o fio de sutura influenciado por vários fatores, dentre eles: seu treinamento ou preferência individual, a característica biológica do material, se absorvível ou inabsorvível; a resistência à tensão de uma sutura, que influencia no tipo e diâmetro do fio a ser escolhido; a localização e o tamanho da incisão ou do campo operatório e as condições particulares do paciente, tais como: idade, peso corporal, outras doenças ou condições clínicas desfavoráveis.

No que se refere a materiais para uso cirúrgico sabe-se que a enfermagem esteve envolvida no preparo de fios e agulhas destinados às suturas desde que os procedimentos cirúrgicos passaram a ser realizados em instituições hospitalares. Atualmente, com a utilização de fios de sutura cirúrgica com propriedades físicas uniformes, desempenho previsível, agulha(s) pré-instalada(s), embalagens apropriadas e já estéreis, a tarefa das enfermeiras de Centro Cirúrgico passa a constituir-se mais em relação à diversidade de opções em fios e agulhas existentes no mercado. Apesar de não ser usuária direta dos fios de sutura cirúrgica, a enfermeira é muitas vezes responsável por escolher os fios que serão comprados, elaborar a estimativa de consumo, providenciar o armazenamento dos fios, assim como a forma de sua distribuição. Para tanto, são seguidas as preferências dos cirurgiões, a rotina de compra estabelecida pelas normas da instituição, a melhor relação custo-benefício, além da disponibilidade no mercado e da influência pela propaganda ${ }^{(7-8)}$.

Castilho, Leite ${ }^{(9)}$ definem previsão de materiais como o levantamento das necessidades da Unidade de Enfermagem, identificando a quantidade e a especificidade dos materiais para suprir essas necessidades. Quanto à provisão, afirmam que consiste na reposição dos materiais, podendo ser realizada conforme o sistema de reposição por tempo, reposição por quantidade ou reposição imediata ao uso.

Testa ${ }^{(10)}$ define estratégia como manobra destinada a ganhar liberdade de ação e pensamento estratégico como um conjunto de comportamentos organizacionais destinados ao manejo de situações para a conquista de um objetivo. Os comportamentos baseados no pensamento estratégico não garantem o alcance da meta estabelecida, mas colocam as condições necessárias para alcançá-la.

Dentro do planejamento estratégico, o fio para sutura cirúrgica pode ser definido como um insumo durável, não-reutilizável, que é consumido na execução da cirurgia, uma atividade de assistência à saúde. Esse objeto está vinculado a processos e faz parte da lógica de programação de uma instituição hospitalar, requerendo duplo manejo: pelo pessoal da administração, no que se refere à obtenção, ao armazenamento e ao fornecimento, e pelo pessoal de saúde, no que diz respeito a sua utilização final.

Assim, o fio de sutura cirúrgica pode ser analisado, por um lado, no ato operatório e, por outro lado, na dinâmica da Unidade de Centro Cirúrgico ou ainda em um contexto mais amplo - a administração do hospital de planejamento estratégico da totalidade dos insumos materiais utilizados na instituição hospitalar. Ou seja, o envolvimento de cada grupo que irá manipular o material é diferente. Para o médico, o enfoque pode estar mais restrito à escolha do fio adequado a cada plano de sutura do procedimento cirúrgico. Para a enfermeira, podemos imaginar que o enfoque muda na medida em que são várias as especialidades e/ou equipes médicas que utilizam o mesmo material, além de ser necessário cuidar do armazenamento e da distribuição. Já para o setor de compras, trata-se de mais um item orçamentário que necessita entrar no processo de aquisição, estocagem e distribuição. Conhecer as questões administrativas da instituição, o material e os usuários finais, interagindo nos processos a eles relacionados pode favorecer o trabalho diário da enfermeira de Centro Cirúrgico.

Apesar de não ser usuária direta dos fios de sutura cirúrgica, a enfermeira é muitas vezes responsável por escolher os fios que serão comprados, elaborar a estimativa de consumo, providenciar o armazenamento dos fios, assim como organizar sua forma de sua distribuição. Para tanto, são seguidas as preferências dos cirurgiões, a rotina de compra estabelecida pelas normas da instituição, a melhor relação custo-benefício, além da disponibilidade no mercado e da influência pela propaganda.
Os fios de sutura cirúrgica e a enfermeira de centro cirúrgico: critérios de previsão e provisão segundo a natureza das instituições hospitalares 
Anita Romano Ribeiro Kazuko Uchikawa Graziano
Considerando-se os aspectos relacionados, este estudo teve como propósito:

- Identificar e descrever os critérios adotados pelas enfermeiras na seleção dos fios de sutura cirúrgica para a elaboração da sua previsão.

- Levantar as modalidades de provisão de fios de sutura cirúrgica adotadas nas Unidades de Centro Cirúrgico.

\section{MATERIAL E MÉTODO}

A pesquisa caracterizou-se como exploratória, descritiva, de campo, transversal, com abordagem quantitativa.

Inicialmente o projeto desta pesquisa foi encaminhado ao Comitê de Ética e Pesquisa da Escola de Enfermagem da Universidade de São Paulo, tendo sido aprovado. A pesquisa desenvolveu-se junto aos Hospitais do Município de São Paulo de porte pequeno (até 51 leitos), médio (51 a 150 leitos), grande (151 a 500 leitos) e especial (acima de 500 leitos), que possuem centro cirúrgico, enfermeira exclusiva para esta unidade e aceitaram participar da pesquisa em resposta à carta convite enviada.

O instrumento de coleta de dados abordou: características da instituição hospitalar, perfil das enfermeiras que participaram da pesquisa e aspectos relacionados aos critérios de previsão e provisão dos fios de sutura. A validação de forma e conteúdo do instrumento de coleta de dados foi obtida por meio da aplicação a um grupo de cinco enfermeiras, com experiência em Centro Cirúrgico e alunas de curso de pós-graduação. Para a coleta dos dados foi utilizada a técnica de entrevista estruturada e semi-estruturada, visando obter informações específicas sobre o problema investigado.

A população alvo deste estudo constituiu-se de 171 hospitais do município de São Paulo obtida na Secretaria de Estado da Saúde de São Paulo em junho de 2000. Fizeram parte as enfermeiras que atenderam aos seguintes critérios de elegibilidade: serem responsáveis (chefes ou substitutas legais) pela Unidade de Centro Cirúrgico; estarem na função há pelo menos um ano; aceitarem voluntariamente fazer parte da pesquisa assinando um termo de consentimento livre e esclarecido.
Do total de 171 hospitais, 27 foram eliminados por serem Casas de Repouso ou hospitais especializados em Psiquiatria. Outros 24 hospitais foram eliminados porque não tinham uma enfermeira específica para a Unidade de Centro Cirúrgico. A população de acesso foi composta por 74 hospitais da rede pública e privada do Município de São Paulo, que atendiam os critérios de elegibilidade. Os dados foram coletados pela autora de janeiro a abril de 2001.

\section{APRESENTAÇÃOE DISCUSSÃO DOS RESULTADOS}

Os dados coletados foram inseridos em um banco de dados eletrônico do Programa EXCEL para tratamento e apresentação. Foi utilizada estatística descritiva para sintetizar e apresentar os dados em tabelas e figuras, utilizando-se freqüência absoluta e percentual. Também foram feitos alguns cruzamentos de interesse, por meio de tabelas de contingência e utilizado o teste de quiquadrado de Pearson, para inferência. Esses testes estatísticos tinham a finalidade de se detectar se havia dependência entre as respostas.

\section{Características das instituições hospitalares}

A natureza da instituição hospitalar é na maioria (68\%) privada, seguida de pública estadual (22\%), pública municipal (9\%) e apenas uma é pública federal. Quanto ao porte do hospital a maioria tem porte médio (54\%), seguido de porte grande (39\%). Das Unidades de Centro Cirúrgico, quase a totalidade ( $80 \%$ ) é geral, apenas $20 \%$ é especializada. Das 15 respostas obtidas para as especialidades na Unidade de Centro Cirúrgico, 40\% é Ginecologia e Obstetrícia, seguida de Cirurgia Pediátrica (27\%) e Oncologia (13\%). As demais especialidades, Cirurgia Cardíaca, Otorrino, dentre outras, participaram igualmente com $7 \%$. O movimento cirúrgico mensal mostra maior concentração nas categorias de 101 a 250 e de 251 a 500 procedimentos anestésico-cirúrgicos por mês, que somados foram responsáveis por $69 \%$. Com relação às modalidades de aquisição de fios de sutura cirúrgica a maioria é feita através de contrato do hospital com fornecedores $(46 \%)$, seguida de concorrência pública (32\%) e cotação mensal, que representou $22 \%$. Esse dado reflete o fato da maioria dos hospitais serem de rede privada. 
Características individuais das enfermeiras que participaram da pesquisa

A maioria das enfermeiras tem mais de 5 anos de formada (84\%). Quanto a cursos de pós-graduação, a maioria das enfermeiras fez especialização na área de Administração Hospitalar (48\%); seguido de Centro Cirúrgico (43\%). Das entrevistadas, a maioria tem de 2 a 5 anos como enfermeiras responsáveis pelas Unidades de Centro Cirúrgico. Chama atenção o grande número de enfermeiras que exerce há pouco tempo o cargo de chefia da Unidade de Centro Cirúrgico, considerando que temos mais 11 entrevistas que foram excluídas do estudo, porque as enfermeiras estão a menos de 1 ano como responsáveis pela unidade. Quanto à participação em alguma sociedade de especialista ou associação houve uma pequena vantagem do $\operatorname{sim}(55 \%)$ sobre o não $(45 \%)$, mas, grande diferença entre as enfermeiras de hospitais públicos e privados. Do total de 41 enfermeiras que fazem parte de uma sociedade, 33 trabalham na rede privada e apenas 8 na rede pública. Todas as enfermeiras que são sócias de sociedades estão associadas a Sociedade Brasileira de Enfermeiros de Centro Cirúrgico (SOBECC) sendo, 61\% apenas à SOBECC, $32 \%$ à SOBECC e Associação Brasileira de Enfermagem (ABEn) e 7\% à SOBECC e Sociedade Brasileira de Gerenciamento (SOBRAGEN). Com relação à abordagem do tema fio de sutura cirúrgica em evento científico, a maioria (84\%) respondeu que o tema não foi abordado em nenhum evento de que participou. $\mathrm{Na}$ questão sobre a situação em que adquiriu a maior parte dos conhecimentos sobre fio de sutura cirúrgica, quase a totalidade (96\%) respondeu que foi na prática diária isoladamente, ou na prática diária e outros meios. Esses dados confirmam o pressuposto de que o tema "fio de sutura cirúrgica" tem sido pouco discutido, apesar do interesse e da valorização do assunto que ficou demonstrado nas entrevistas realizadas para este trabalho.

\section{Aspectos relacionados aos fios de sutura cirúrgica}

Os aspectos relacionados aos fios de sutura cirúrgica que mais interessam às enfermeiras que responderam a pesquisa dizem respeito à aplicação/indicação dos fios em geral $(35 \%)$, seguido de características de cada fio e de critérios de substituição, ambos com 26\%. Quanto aos fios disponíveis na
Unidade de Centro Cirúrgico apenas uma das entrevistadas não citou os fios Catgut Simples, Catgut Cromado e Vicryl. Todos $(100 \%)$ citaram o fio de Poliamida. Responderam todos, menos o Ácido Poliglicólico 16\% das entrevistadas, enquanto $12 \%$ responderam todos, menos a Polidioxanona e o Ácido Poliglicólico. A maioria dos hospitais possui a maioria dos fios de sutura cirúrgica disponíveis no mercado em variedade de composição, tanto absorvíveis, quanto inabsorvíveis. Com relação aos critérios para seleção dos fios de sutura cirúrgica, a maioria (64\%) foi por solicitação individual do cirurgião, sendo $28 \%$ isoladamente, $28 \%$ juntamente com padronização por especialidades e $8 \%$ com demais critérios. Padronização por especialidades, isoladamente, foi responsável por $34 \%$ das respostas. No que diz respeito aos meios de provisão dos fios de sutura cirúrgica a maioria das respostas foi para estoque no Centro Cirúrgico (40\%) seguida de kit por procedimento ou especialidade (32\%). O estoque principal de fios localiza-se em sua maioria no almoxarifado do hospital (77\%) seguido de estoque no Centro Cirúrgico (18\%). A aquisição de fios de sutura cirúrgica é feita em sua maioria por contrato com fornecedores $(46 \%)$, seguida de concorrência pública (32\%) e de cotação mensal (22\%). Os procedimentos para provisão de fios no hospital são na maioria por reposição da cota estabelecida (58\%), seguida de contagem periódica (38\%). Quanto aos critérios para exclusão de um fio de sutura cirúrgica, a baixa frequiência de uso foi responsável pela maioria das respostas (76\%), sendo que dessas, $27 \%$ foi de baixa freqüência de uso e solicitação de substituição por parte do cirurgião. No que se relaciona aos meios para conhecer novas opções em fios de sutura cirúrgica o contato com representante de fabricantes esteve presente em $78 \%$, isoladamente ou junto com outras opções. A opção através dos cirurgiões apareceu em $58 \%$ das respostas, também isoladamente ou junto com outras. O conjunto dessas duas foi responsável por $28 \%$ das respostas, enquanto que a opção contato com representante do fabricante isoladamente em $22 \%$. Com relação aos últimos fios de sutura cirúrgica que passaram a ser adquiridos pelo hospital, o Monocryl (Poliglecaprone) foi responsável por $45 \%$, enquanto o PDS (Polidioxanona) por $39 \%$. Os demais se diluíram. Deve ser observado que apenas 33 pessoas responderam a essa questão. Quanto ao solicitante, a maio-
Os fios de sutura cirúrgica e a enfermeira de centro cirúrgico: critérios de previsão e provisão segundo a natureza das instituições hospitalares

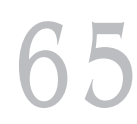

Rev Esc Enferm USP 2003; 37(4):61-8. 
Anita Romano Ribeiro Kazuko Uchikawa Graziano ria dos que responderam, declararam ter sido a Cirurgia Geral (41\%) seguido da Cirurgia Urológica (28\%) e da Cirurgia Plástica (25\%).

A Tabela 1 mostra as freqüências observadas para o cruzamento de natureza da insti- tuição hospitalar com modalidade de aquisição dos fios de sutura cirúrgica, procedimentos para provisão, formas de seleção e tipos de fios, valor do qui-quadrado observado e a respectiva significância estatística.

Tabela 1 - Freqüências observadas para o cruzamento da natureza da instituição com: modalidade de aquisição, procedimentos para provisão, seleção de fios, tipo de fio e valor do qui-quadrado observado e respectiva significância estatística (São Paulo, 2001)

\begin{tabular}{|c|c|c|c|c|}
\hline & \multicolumn{2}{|c|}{ Natureza } & \multirow[t]{2}{*}{ Total } & \multirow[t]{2}{*}{$\mathbf{x} 2$} \\
\hline & Privada & Pública & & \\
\hline \multicolumn{5}{|l|}{ Modalidade de Aquisição } \\
\hline $\begin{array}{c}\text { Contrato do hospital com fornecedor(es) } \\
\text { Cotação mensal } \\
\text { Concorrência Pública }\end{array}$ & $\begin{array}{c}34 \\
15 \\
1\end{array}$ & $\begin{array}{c}0 \\
1 \\
23\end{array}$ & $\begin{array}{l}34 \\
16 \\
24\end{array}$ & \\
\hline Total & 50 & 24 & 74 & $65,35^{* *}$ \\
\hline \multicolumn{5}{|l|}{ Procedimentos para provisão } \\
\hline $\begin{array}{c}\text { Contagem periódica do estoque/tempo } \\
\text { Reposição da cota estabelecida/quant. } \\
\text { Reposição através do débito na ficha da sala de } \\
\text { operações } \\
\text { Consignação }\end{array}$ & $\begin{array}{c}8 \\
39 \\
2 \\
1\end{array}$ & $\begin{array}{c}20 \\
4 \\
0 \\
0\end{array}$ & $\begin{array}{l}28 \\
43 \\
2 \\
1\end{array}$ & \\
\hline Total & 50 & 24 & 74 & $31,37 * *$ \\
\hline \multicolumn{5}{|l|}{ Seleção dos fios } \\
\hline Solicitação individual do cirurgião & 16 & 5 & 21 & \\
\hline Padronização por especialidades & 13 & 12 & 25 & \\
\hline Padronização de grupo de compras & 1 & 0 & 1 & \\
\hline Padronização do hospital & 0 & 1 & 1 & \\
\hline $\begin{array}{c}\text { Solicitação individual do cirurgião e Padronização } \\
\text { por especialidades }\end{array}$ & 17 & 4 & 21 & \\
\hline $\begin{array}{l}\text { Solicitação individual do cirurgião, Padronização } \\
\text { por especialidades e Padronização do hospital }\end{array}$ & 1 & 0 & 1 & \\
\hline $\begin{array}{c}\text { Solicitação individual do cirurgião e Padronização } \\
\text { de grupo de compras }\end{array}$ & 1 & 0 & 1 & \\
\hline $\begin{array}{c}\text { Solicitação individual do cirurgião, Padronização } \\
\text { de grupo de compras e Iniciativa da enfermeira } \\
\text { de centro cirúrgico }\end{array}$ & 1 & 0 & 1 & \\
\hline $\begin{array}{l}\text { Solicitação individual do cirurgião e Iniciativa da } \\
\text { enfermeira de centro cirúrgico }\end{array}$ & 0 & 1 & 1 & \\
\hline $\begin{array}{l}\text { Solicitação individual do cirurgião e Padronização } \\
\text { do hospital }\end{array}$ & 0 & 1 & 1 & \\
\hline Total & 50 & 24 & 74 & $11,28 \mathrm{~ns}$ \\
\hline
\end{tabular}


Observa-se que houve significância estatística de dependência entre natureza da instituição com todas as variáveis cruzadas na Tabela 1, exceção feita à seleção dos fios de sutura cujo cruzamento foi não significativo pelo teste de qui-quadrado:

- quando se cruzou natureza da instituição hospitalar com modalidade de aquisição de fios pôde se observar que a maioria das instituições privadas adquire os fios por contrato do hospital com fornecedores, seguido de cotação mensal, enquanto nas instituições públicas por concorrência pública (convite, tomada de preços ou registro de preços). Essa dependência entre as duas variáveis foi significativa ao nível de $1 \%$.

- quando se cruzou natureza da instituição hospitalar com procedimentos para provisão evidenciou-se que nas privadas a maior freqüência foi para reposição da cota estabelecida, ou seja, a reposição é por quantidade mantendo-se sempre a cota estabelecida como estoque do hospital. $\mathrm{Na}$ pública a maior freqüência foi para contagem periódica do estoque e reposição, ou seja, a reposição é por tempo estabelecido mostrando que existe um estabelecimento de tempo entre as concorrências públicas. O teste de qui-quadrado mostrou também aqui dependência significativa ao nível de $1 \%$ entre as variáveis.

Retomando Testa ${ }^{(10)}$ e seguindo a sua definição de planejamento estratégico conseguimos identificar três lógicas distintas que envolvem os fios de sutura cirúrgica. A lógica da administração, que se refere à obtenção, armazenamento e fornecimento às unidades hospitalares; a lógica do cirurgião, direcionada à escolha do fio de sutura cirúrgica mais adequado ao paciente; e a lógica da enfermeira de Centro Cirúrgico, que envolve previsão, provisão, organização, controle e disponibilização do material. Por essa razão consideramos a enfermeira um elo importante entre a administração e o usuário final, sendo freqüentemente responsável por atender as expectativas dos dois lados. Nesse sentido, o domínio de informações técnicas, o acesso a lançamentos ou opções diferentes da já padronizada na instituição, além de subsídios para realizar as substituições que se fizerem necessárias entre marcas e/ou tipos de fios, e o conhecimento da indicação de uso de cada fio conforme sua composição, assim como, a atuação da enfermeira nos processos administrativos que envolvem os fios de sutura cirúrgica, tudo isso, forma um conjunto que contribui para a solução de conflitos surgidos no dia-a-dia. Cada elemento cumprindo bem sua tarefa dentro da lógica do planejamento estratégico favorece um ambiente de trabalho harmonioso e garante uma assistência de qualidade ao paciente cirúrgico.

\section{CONCLUSÕESE CONSIDERAÇÕES FINAIS}

Quanto às características das instituições hospitalares que participaram do estudo, a maioria é da rede privada (68\%), de porte médio (54\%), com Unidade de Centro Cirúrgico geral $(80 \%)$ e movimento de até 500 procedimentos mensais $(69 \%)$. Nesses hospitais a solicitação do cirurgião é o fator mais importante como critério de seleção dos fíos, aparecendo em 36 das 50 respostas para essa questão, seja isoladamente ou, acompanhada de padronização por especialidade. Em contraposição, nos hospitais da rede pública a padronização dos fios é mais freqüen-temente estabelecida por cada especialidade. Solicitação do cirurgião e padronização por especialidade separadamente aparecem em seguida.

Quanto aos fios de sutura cirúrgica, os aspectos que mais interessam às enfermeiras estão relacionados à aplicação/indicação, características dos fios e critérios de substituição. Das enfermeiras que participaram da entrevista, 96\% declararam ter adquirido a maior parte dos conhecimentos sobre fios na prática diária, isoladamente, ou acompanhado de outros meios. Dos fios disponíveis, 64\% deles são padronizados por solicitação do cirurgião. Dos meios para provisão de fios na sala de operações, $40 \%$ dos fios são escolhidos no momento da cirurgia, enquanto $32 \%$ são kits por especialidade. No hospital, 46\% são adquiridos do fornecedor, $32 \%$ através de concorrência pública. O estoque é reposto segundo cota estabelecida em $58 \%$ dos hospitais, o que mantém relação com a natureza privada da instituição, significativo ao nível de $1 \%$. Nas instituições públicas predomina provisão por meio de contagem periódica do estoque. A variedade de fios também está relacionada à natureza, havendo maior variedade de fios em hospitais privados, e uma inconstância nos públicos, significativo ao nível de 5\%. A maioria dos hospitais pos-
Os fios de sutura cirúrgica e a enfermeira de centro cirúrgico: critérios de previsão e provisão segundo a natureza das

instituições hospitalares 
Anita Romano Ribeiro Kazuko Uchikawa Graziano
Recebido: 20/11/2002 Aprovado: 27/08/2003

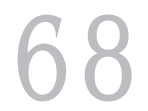

Rev Esc Enferm USP 2003; 37(4): 61-8. sui a maioria dos fios de sutura cirúrgica disponíveis no mercado em variedade de composição, tanto na categoria de absorvíveis, quanto inabsorvíveis. Apenas uma das entrevistadas não citou os fios Catgut Simples, Catgut Cromado e Vicryl. Os demais citaram esses fios, entre outros. Todos (100\%) citaram o fio de Poliamida. Observa-se também que $16 \%$ responderam que usam todos, menos o Ácido Poliglicólico, $12 \%$ responderam que usam todos menos o Polidioxanona e o Ácido Poliglicólico.

A enfermagem esteve envolvida no preparo de fios e agulhas destinados às suturas, desde que os procedimentos cirúrgicos passaram a ser realizados em instituições hospitalares. Atualmente, com a utilização de fios de sutura cirúrgica de pronto-uso, com agulhas pré-instaladas, em embalagens apropriadas e já estéreis, a tarefa das enfermeiras tem mais relação com a diversidade de opções existentes no mercado. Na vivência profissional em Unidades de Centro Cirúrgico, observamos que a tarefa de disponibilizar os fios de sutura cirúrgica é muitas vezes motivo de tensão por envolver conhecimento técnico, autonomia, fluxo de decisões, a natureza pública ou privada da administração e a própria rotina da instituição hospitalar.

Blust $^{(7)}$ e Zokal $^{(8)}$ advertem sobre a necessidade de reduzir gastos com os fios de sutura cirúrgica e resolver questões relacionadas a inventário - como a variedade e a quantidade -, mantendo a qualidade da assistência prestada ao paciente $\mathrm{e} o$ atendimento às necessidades e às preferências dos cirurgiões. Constatamos

\section{REFERÊNCIAS}

(1) Goffi FS, Tolosa EMC. Operações fundamentais. In: Goffi FS. Técnica Cirúrgica: bases anatômicas, fisiopatológicas e técnicas cirúrgicas. 4a ed. São Paulo: Atheneu; 1996. p. $52-3$

(2) Makenzie D. The history of sutures. Med Hist 1973; 4:158-68.

(3) Farmacopéia brasileira. $4^{a}$ ed. São Paulo: Atheneu; 1988.

(4) Associação Brasileira de Normas Técnicas. NBR 13904 - Fios para sutura cirúrgica. Rio de Janeiro; 2003.

(5) Hering FLO, Gabor S, Rosenberg D. Bases técnicas e teóricas de fios e suturas. São Paulo: Roca; 1993. que existe pouco subsídio na literatura científica brasileira sobre materiais de síntese cirúrgica para fundamentar critérios de determinação da variedade dos itens em fios de sutura cirúrgica quanto à composição do fio, diâmetros e agulha(s), da quantidade necessária por procedimento, além de substituição quando necessário. A complexidade crescente dos procedimentos cirúrgicos, seu custo e a variedade dos fios de sutura e das marcas disponíveis no mercado tornam necessária a racionalização do inventário e da distribuição, para a redução de despesas e do tempo gasto em atividades não relacionadas à assistência direta ao paciente.

A realização deste estudo mostrou que as enfermeiras têm interesse em conhecer mais sobre fios de sutura cirúrgica para melhor direcionar suas atividades de previsão e provisão. Nossa hipótese inicial de que o assunto tem lacunas de conhecimento entre as enfermeiras de Centro Cirúrgico foi confirmada. Em várias entrevistas elas mostravam-se receosas em participar, inclusive verbalizando que poderiam não ter conhecimento suficiente para responder as questões a serem feitas. Foi freqüente o número de enfermeiras que ressaltou a importância do conhecimento sobre fios de sutura cirúrgica para a prática diária.

Esperamos ter contribuído para reflexões sobre o tema e até mesmo para a busca de soluções para dificuldades identificadas nas atividades que permeiam a tarefa de disponibilizar os fios de sutura cirúrgica, uma das várias atribuições das enfermeiras de Centro Cirúrgico.
(6) Benicewicz BC, Hopper PK. Polymers for absorbable surgical sutures Part I. J Bioact Compat Polymers 1990; 5:453:72.

(7) Blust JB, Avigne GA, Miller BE. Suture conversion without the knots. AORN J 1997; 65(1):116-17.

(8) Zokal F. Made-to-order suture packs. AORN J 1990; 51(3): 817-27.

(9) Castilho V, Leite MMJ. A administração de recursos humanos na enfermagem. In: Kurcgant P. Administração em enfermagem. São Paulo: EPU; 1991. p. 73-88.

(10)Testa M. Pensamento estratégico e a lógica de programação: o caso da saúde. São Paulo: HUCITEC; 1995. 